Bulletin of the Section of Logic

Volume 47/2 (2018), pp. 129-139

http://dx.doi.org/10.18778/0138-0680.47.2.05

Michał M. Stronkowski

\title{
GRZEGORCZYK ALGEBRAS REVISITED
}

\begin{abstract}
We provide simple algebraic proofs of two important facts, due to Zakharyaschev and Esakia, about Grzegorczyk algebras.

Keywords: Grzegorczyk algebras, free Boolean extensions of Heyting algebras, stable homomorphisms.

2010 Mathematics Subject Classification. 03G25, 06E25, 06D20, 03B45.
\end{abstract}

\section{Introduction}

Let us consider the modal formula

$$
\operatorname{grz}=\square(\square(p \rightarrow \square p) \rightarrow p) \rightarrow p .
$$

Let GRZ be the normal extension of S4 modal logic axiomatized by grz [11]. Let INT be the intuitionistic logic. The importance of GRZ follows from the fact that it is a largest normal extension of S4 into which INT embeds via the Gödel-Tarski translation. In fact much more is true. The celebrated Blok-Esakia theorem states that there is one to one correspondence between the extensions of INT and the normal extensions of GRZ. Moreover, this correspondence is given, in some strict sense, by the GödelTarski translation $[6,15,18]$.

This paper grew out of work on algebraic approach to the Blok-Esakia theorem for universal classes [17]. Grzegorczyk algebras play a prominent role there. They form a variety which gives an algebraic semantics for GRZ. Here we presents new, simple and purely algebraic proofs of two important fact about them. The first, proved by Zakharyaschev in [19], states that an 
interior algebra is a Grzegorczyk algebra iff its dual descriptive frame is not subreducible to a two element cluster (Theorem 2.4). The second result is due to Esakia [8]. It characterizes the free Boolean extensions of Heyting algebras as those interior algebras which do not admit a stable homomorphism onto a four element simple interior algebra (the dual algebra of a two element cluster) (Theorem 3.2). This fact was proved with the use of Esakia duality for interior algebras. The free Boolean extensions of Heyting algebras generate the variety of Grzegorczyk algebras. They are the key objects in the algebraic proof of the Blok-Esakia theorem [3, 17, 18].

\section{Induced stable homomorphisms}

We assume that the reader is familiar with basic properties of Boolean algebras [10]. An interior algebra (aka closure algebra and topological Boolean algebra) is a Boolean algebra endowed with an additional unary operation $\square$ such that for all its elements $a, b$ we have $\square(a \wedge b)=\square a \wedge \square b$, $\square 1=1$ and $\square \square a=\square a \leqslant a$. Let $\diamond a=\neg \square \neg a$. An element $a$ of an interior algebra $\mathbf{M}$ is open if $\square a=a$ and closed if $a=\diamond a$. For an interior algebra there is one to one correspondence between its congruences and its open filters, i.e., Boolean filters closed under $\square$ operation. It is given by $\theta \mapsto 1 / \theta$ and $F \mapsto \theta_{F}$, where $(a, b) \in \theta_{F}$ iff $a \leftrightarrow b \in F$ (here $\theta$ is a congruence and $F$ is an open filter). For an interior algebra, an element $b$ belongs to the open filter generated by $a$ iff $\square a \leqslant b$. It follows that an interior algebra is subdirectly irreducible iff it has a largest non-top open element. And an interior algebra is simple iff it has exactly two open elements 0 and 1. A simple four-element interior algebra will be denoted by $\mathbf{S}_{2}$.

An interior algebra $\mathbf{M}$ is a Grzegorczyk algebra if it satisfies

$$
\square(\square(a \rightarrow \square a) \rightarrow a) \leqslant a
$$

for every $a \in M[8]$. Note that in every modal algebra satisfying the reflexivity condition $\square x \leqslant x$ we have $\square(a \rightarrow \square a) \leqslant a \rightarrow \square a$. Hence also $a-\square a \leqslant \neg \square(a \rightarrow \square a)$. Thus

$$
a \vee \neg \square(a \rightarrow \square a)=\square a \vee(a-\square a) \vee \neg \square(a \rightarrow \square a)=\square a \vee \neg \square(a \rightarrow \square a)
$$

It follows that an interior algebra is a Grzegorczyk algebra iff

$$
\square(\square(a \rightarrow \square a) \rightarrow \square a) \leqslant \square a
$$

holds for every $a \in M$ (this alternative axiomatization may be found in $[3])$. 
Let us recall the notion of stable homomorphisms. Let $\mathbf{M}$ and $\mathbf{N}$ be interior algebras. We say that a mapping $f: M \rightarrow N$ is a stable homomorphism from $\mathbf{M}$ into $\mathbf{N}$ if it is a Boolean homomorphism and

$$
f(\square a) \leqslant \square f(a)
$$

holds for every $a \in M$. In such a case, we write $f: \mathbf{M} \rightarrow_{s} \mathbf{N}$. The reader may consult e.g. [2,9] for the importance of stable homomorphisms in modal logic (in [9] they are called continuous morphism). Note that a stable homomorphism does not need to be a homomorphism. For instance $\mathbf{S}_{2}$ does not admit a homomorphism onto a two-element interior algebra, but it admits such stable homomorphisms.

LEMMA 2.1. Let $\mathbf{M}$ be an interior algebra and $\mathbf{S}$ be a simple interior algebra. Then the mapping $f: M \rightarrow S$ is a stable homomorphism if and only if it is a Boolean homomorphism and for every $a \in M$ we have $f(\square a) \in\{0,1\}$.

Proof: Assume that $f$ is a stable homomorphism. By the definition, $f$ is a Boolean homomorphism. Moreover, if $f(\square a)<1$ then $f(\square a)=$ $f(\square \square a) \leqslant \square f(\square a)=0$. For the opposite implication assume that $f$ is a Boolean homomorphism and $f(\square a)=\{0,1\}$. If $f(\square a)=0$ then, clearly, $f(\square a) \leqslant \square f(a)$. If $f(\square a)=1$ then, since $\square a \leqslant a, f(a)=1$, and so $f(\square a)=1=\square f(a)$.

We say that a (stable) homomorphism $f$ from $\mathbf{M}$ into $\mathbf{N}$ is principal if the Boolean filter $f^{-1}(1)$ is principal. This means that there is $d \in M$ such that $f(a)=1$ iff $d \leqslant a$ and $f(a)=0$ iff $a \leqslant \neg d$ for all $a \in M$. Let $f: \mathbf{M} \rightarrow_{s} \mathbf{N}$ be a surjective principal stable homomorphism and assume that the filter $f^{-1}(1)$ is generated by $d$. Then for every $a \in M$ we have $f(a)=f(a \vee \neg d)$, and hence the inequality

$$
f(\square(a \vee \neg d)) \leqslant \square f(a)
$$

holds. Let us say that $f$ is induced if we have the equality

$$
\square f(a)=f(\square(a \vee \neg d))
$$

for all $a \in M$. Note that then

$$
\diamond f(a)=f(\diamond(a \wedge d)) .
$$

With every element $d \in M$ we may associate an induced surjective stable homomorphism $f_{d}: \mathbf{M} \rightarrow_{s} \mathbf{M}_{d}$ such that $f_{d}^{-1}(1)$ is a filter generated by $d$. 
Here $f_{d}$ is essentially unique, i.e., it is unique up to isomorphism of $\mathbf{M}_{d}$. (We may also define it in a more explicit way. E.g., let the carrier $M_{d}$ be $\{a \in M \mid a \leqslant d\}$, the join, the meet of $a, b$ and the 0 in $\mathbf{M}_{d}$ be the same as in $\mathbf{M}$, the 1 in $\mathbf{M}_{d}$ be $d$, the negation of $a$ in $\mathbf{M}_{d}$ be given by $\neg a \wedge d$, and the box of $a$ be given by $\square(a \vee \neg d) \wedge d$. Then $f_{d}(a)=a \wedge d$. In [1] the algebra $\mathbf{M}_{d}$ is called the relativisation of $\mathbf{M}$ to $d$.)

The following lemma is generally known. The reason why we provide the proof here is to emphasize the condition (3).

LEMma 2.2. Let $\mathbf{M}$ be an interior algebras. Then the following conditions are equivalent:

1. $\mathbf{M}$ is a McKinsey algebra, i.e., $\mathbf{M} \models \forall x \square \diamond x \leqslant \diamond \square x$,

2. $\mathbf{M} \models \forall x \square x=0 \Rightarrow \square \diamond x=0$,

3. $\mathbf{M} \models \forall x \square \diamond(x-\square x)=0$,

4. $\mathbf{M}$ does not admit a (principal) homomorphism onto $\mathbf{N}$ such that $\mathbf{S}_{2} \leqslant \mathbf{N}$.

PROOF:

$(1) \Rightarrow(2)$ Assume that in $\mathbf{M}$ we have $\square a=0$. Then $\diamond \square a=\diamond 0=0$, and by (1), $\square \diamond a=0$.

$(2) \Rightarrow(3)$ Let $a \in M$. By the monotonicity of the $\square$ operation, $\square(a-\square a) \leqslant$ $\square a$. By the reflexivity, $\square(a-\square a) \leqslant a-\square a$. Hence $\square(a-\square a)=0$, and by $(2), \square \diamond(a-\square a)=0$.

$\sim(4) \Rightarrow \sim(3)$ Let $h: \mathbf{M} \rightarrow \mathbf{N}$ be a surjective homomorphism and $a \in M$ be such that $h(a)$ is a (co)atom in $\mathbf{S}_{2}$. Then $h(\square a)=\square h(a)=0$ and $h(a-\square a)=h(a)$. Hence $h(\square \diamond(a-\square a))=\square \diamond h(a)=\square 1=1$. It follows that $\square \diamond(a-\square a) \neq 0$.

$\sim(1) \Rightarrow \sim(4)$ Assume that $\square \diamond a \nless \nless \square a$ for some $a \in M$. Let $F$ be the (open) filter of $\mathbf{M}$ generated by $\square \diamond a \wedge \square \diamond \neg a$. The assumption guaranties that $F$ is proper, i.e., $F \neq M$. In $\mathbf{M} / F$ we have $\diamond a / F=1$ and $\diamond \neg a / F=1$. Hence the subalgebra of $\mathbf{M} / F$ generated by $a / F$ is isomorphic to $\mathbf{S}_{2}$. (At this point the transitivity of $\mathbf{M}$ is used.)

Lemma 2.3. Let $\mathbf{M}$ be an interior algebra and $a, d \in M$. Then in $\mathbf{M}$ we have $\square(\square(a \rightarrow \neg d) \rightarrow \neg d) \leqslant \neg d$ if and only if in $\mathbf{M}_{d}$ we have $\square \diamond f_{d}(a)=0$. In particular, $\mathbf{M}$ satisfies (Grz ${ }^{\square}$ ) for $a$ if and only if in $\mathbf{M}_{\neg \square a}$ we have $\square \diamond f_{\neg \square a}(a)=0$. 
Proof: By the definition of $f_{d}$, we have

$$
\begin{aligned}
\square \diamond f_{d}(a) & =f_{d}(\square(\diamond(a \wedge d) \vee \neg d)) \\
& =f_{d}(\square(\square(a \rightarrow \neg d) \rightarrow \neg d)) .
\end{aligned}
$$

Thus

$$
\square \diamond f_{d}(a)=0 \quad \text { iff } \quad \square(\square(a \rightarrow \neg d) \rightarrow \neg d) \leqslant \neg d .
$$

We obtain the second statement by substituting $d=\neg \square a$.

The equivalence $(1) \Leftrightarrow(4)$ in the following theorem was obtained by Zakharyaschev in [19, Example 5] (see [5, Proposition 9.3] for the full proof). It is expressed there in the dual language (in the sense of [5, Theorem 8.24]) with the use of general frames and subreductions.

THEOREM 2.4. Let $\mathbf{M}$ be an interior algebra. Then the following conditions are equivalent:

1. $\mathbf{M}$ is a Grzegorczyk algebra,

2. for every closed $d \in M, \mathbf{M}_{d}$ is a McKinsey algebra,

3. for every $d \in M, \mathbf{M}_{d}$ is a McKinsey algebra.

4. there is no induced stable homomorphism $f: \mathbf{M} \rightarrow_{s} \mathbf{N}$ such that $\mathbf{S}_{2} \leqslant$ $\mathrm{N}$.

5. there is no surjective principal stable homomorphism $f: \mathbf{M} \rightarrow_{s} \mathbf{N}$ such that $\mathbf{S}_{2} \leqslant \mathbf{N}$.

Proof: The implications $(5) \Rightarrow(4)$ and $(3) \Rightarrow(2)$ are obvious.

$\sim(3) \Rightarrow \sim(4)$ Assume that $\mathbf{M}_{d}$ is not a McKinsey algebra for some $d \in$ $M$. Then, by Lemma 2.2 (the implication $(4) \Rightarrow(1)$ ), there is a principal surjective homomorphism $h: \mathbf{M}_{d} \rightarrow \mathbf{N}$ such that $\mathbf{S}_{2} \leqslant \mathbf{N}$. Then $h \circ f_{d}$ is an induced stable homomorphism onto $\mathbf{N}$.

$\sim(1) \Rightarrow \sim(2)$ Assume that $\left(\mathrm{Grz}^{\square}\right)$ fails for $a$ in $M$. By Lemma 2.3, in $\mathbf{M}_{\neg \square a}$ we have $\square \diamond f_{\neg \square a}(a)>0$. But, since $\square a \leqslant a$, in $\mathbf{M}_{\neg \square a}$ we also have

$$
\square f_{\neg \square a}(a)=f_{\neg \square a}(\square(a \vee \square a))=f_{\neg \square a}(\square a)=0 .
$$

Thus, by Lemma 2.2 (the implication $(1) \Rightarrow(2)$ ), $\mathbf{M}_{\neg \square a}$ is not a McKinsey algebra.

$\sim(5) \Rightarrow \sim(1)$ Let $f: \mathbf{M} \rightarrow_{s} \mathbf{N}$ be a surjective principal stable homomorphism $f: \mathbf{M} \rightarrow \mathbf{N}$ such that $\mathbf{S}_{2} \leqslant \mathbf{N}$. Let $d$ be a generator of the Boolean filter $f^{-1}(1)$. Let $c$ be a (co)atom in $\mathbf{S}_{2}$ and $b \in M$ be such that $f(b)=c$. 
Put $a=b \vee \neg d$. Then $f(a)=f(b) \vee \neg f(p)=c$. Let us show that (Grz) fails for $a$. Since $f$ is a stable homomorphism, $f(\square a) \leqslant \square f(a)=0$. Thus $f(a \rightarrow \square a)=f(a) \rightarrow 0=\neg f(a)=\neg c$. Similarly $f(\square(a \rightarrow \square a))=0$. Hence, $\square(a \rightarrow \square a) \leqslant \neg d<a$, and thus $\square(\square(a \rightarrow \square a) \rightarrow a)=1 \nless a$. (Note that $\square(a \rightarrow \square a) \leqslant \square a$ and $\square(\square(a \rightarrow \square a) \rightarrow \square a)=1 \nless \square a$. Hence clearly $\left(\mathrm{Grz}^{\square}\right)$ also fails for $a$.)

\section{Free Boolean extensions of Heyting algebras}

The connection of Heyting algebras with interior algebras is given by the following McKinsey-Tarski theorem [14, Section 1] (see also [3, Chapter 1], [4, Theorem 2.2] and [13, Section 3]). Recall that open elements of an interior algebra $\mathbf{M}$ form the Heyting algebra $\mathbf{O}(\mathbf{M})$ with the order structure inherited from $\mathbf{M}$.

TheOREM 3.1. For every Heyting algebra $\mathbf{H}$ there is an interior algebra $\mathrm{B}(\mathbf{H})$ such that

1. $\mathrm{OB}(\mathbf{H})=\mathbf{H}$;

2. for every interior algebra $\mathbf{M}$, if $\mathbf{H} \leqslant \mathrm{O}(\mathbf{M})$, then $\mathbf{B}(\mathbf{H})$ is isomorphic to the subalgebra of $\mathbf{M}$ generated by $H$.

The algebra $\mathrm{B}(\mathbf{H})$ is called the free Boolean extension of $\mathbf{H}$. Note that Theorem 3.1 immediately yields that an interior algebra is isomorphic to a free Boolean extension of a Heyting algebra iff it is generated by its open elements.

Esakia developed duality theory for interior algebras [7, 8]. In particular, he provided a characterization of free Boolean extensions of Heyting algebras. It says that an interior algebra is isomorphic to a free Boolean extension of a Heyting algebras if and only if its dual Esakia space has no non-trivial clusters [8, Theorem 12.7]. In algebraic terms it may be formulated as follows.

THEOREM 3.2. An interior algebra is isomorphic to a free Boolean extension of a Heyting algebra if and only if it does not admit a stable homomorphism onto $\mathbf{S}_{2}$.

The proof will follow from some known extensions of the prime filter theorem for Boolean algebras. 
Lemma 3.3. Let $\mathbf{C}$ and $\mathbf{D}$ be Boolean algebras, $\mathbf{D}$ be a subalgebra of $\mathbf{C}$ and $U$ be an ultrafilter of $\mathbf{D}$. Then there exists an ultrafilter $W$ of $\mathbf{C}$ such that $W \cap D=U$.

Proof: Let $F=\{c \in C \mid(\exists d \in U) d \leqslant c\}$. Then $F$ is a proper filter of $\mathbf{C}$. By the prime filter theorem, there exists an ultrafilter $W$ of $\mathbf{C}$ extending $F$. Then $W \cap D$ is an ultrafilter of $\mathbf{D}$ extending $U$. By the maximality of $U$ (as a proper filter of $\mathbf{D}$ ), we have $W \cap D=U$.

The following less obvious lemma may be found, as a harder exercise, in [10, Exercise 15 in Chapter 20]. Its proof may be found in [12]. We provide a more direct one. (Actually, the formulation in [12] is slightly stronger. Our proof yields also this extension.) It also clarifies that it is enough to assume prime filter theorem for Boolean algebras and the full strength of the axiom of choice is not needed here.

Lemma 3.4. Let $\mathbf{B}$ be a proper Boolean subalgebra of $\mathbf{A}$. Then there are two distinct ultrafilters $U_{1}$ and $U_{2}$ of $\mathbf{A}$ such that $U_{1} \cap B=U_{2} \cap B$.

Proof: It is convenient to formulate the proof with the use of homomorphisms. Recall that ultrafilters of Boolean algebras are exactly preimages of 1 for homomorphisms onto a two-element Boolean algebra 2.

Let $e \in A-B$. Let $G_{1}$ and $G_{2}$ be two different proper filters of $\mathbf{A}$ such that $e \in G_{1}, \neg e \in G_{2}$ and

$$
G_{1} \cap B=G_{2} \cap B=G .
$$

For instance, we may take

$$
\begin{aligned}
& G_{1}=\left\{a_{1} \wedge a_{2} \in A \mid e \leqslant a_{1} \text { and }(\exists b \in B) \neg e<b \leqslant a_{2}\right\}, \\
& G_{2}=\left\{a_{1} \wedge a_{2} \in A \mid(\exists b \in B) e<b \leqslant a_{1} \text { and } \neg e \leqslant a_{2}\right\} .
\end{aligned}
$$

Since $e$ and $\neg e$ do not belong to $B$ and $B$ is closed under the $\wedge$ operation, such defined $G_{1}$ and $G_{2}$ are indeed filters of $\mathbf{A}$. Moreover, $e \in G_{1}$, $\neg e \in G_{2}$. Furthermore, if $a_{1} \geqslant e$ and $a_{2}>\neg e$, then $a_{1} \wedge a_{2}>0$. This shows that $G_{1}$ is proper. Similarly we infer that $G_{2}$ is proper. Also it is the case that $G_{1} \cap B=G_{2} \cap B$. Indeed, if $e \leqslant a_{1}, \neg e<b \in B, b \leqslant a_{2}$ and $a_{1} \wedge a_{2} \in B$ then $\left(a_{1} \wedge a_{2}\right) \vee \neg b=\left(a_{1} \vee \neg b\right) \wedge\left(a_{2} \vee \neg b\right)=a_{1} \wedge 1=a_{1}$, and hence $a_{1} \in B$. This shows that $G_{1} \cap B \subseteq G_{2}$. Similarly, one may show that $G_{2} \cap B \subseteq G_{1}$.

Let $l: \mathbf{B} \rightarrow \mathbf{A}$ be the embedding. Let us also define $f_{i}: \mathbf{A} \rightarrow \mathbf{A} / G_{i} ; a \mapsto$ $a / G_{i}$, and $f: \mathbf{B} \rightarrow \mathbf{B} / G ; b \rightarrow b / G$. Since $G_{i} \cap B=G$, the homomorphisms 
$k_{i}: \mathbf{B} / G \rightarrow \mathbf{A} / G_{i} ; b / G \rightarrow b / G_{i}$ are well defined and injective. We have the following commutative diagrams.

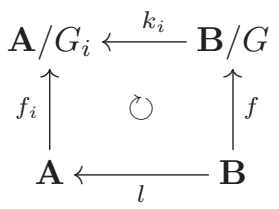

By the prime filter theorem, there exists a homomorphism $g: \mathbf{B} / G \rightarrow \mathbf{2}$. By Lemma 3.3, there are surjective homomorphisms $g_{i}: \mathbf{A} / G_{i} \rightarrow \mathbf{2}$ such that $g=g_{i} \circ k_{i}$. Let

$$
U_{i}=\left(g_{i} \circ f_{i}\right)^{-1}(1) .
$$

Since $G_{i} \subseteq U_{i}$, we have $e \in U_{1}$ and $\neg e \in U_{2}$. This yields that $U_{1} \neq U_{2}$. Furthermore, we have

$$
(g \circ f)^{-1}(1)=\left(g_{i} \circ k_{i} \circ f\right)^{-1}(1)=\left(g_{i} \circ f_{i} \circ l\right)^{-1}(1)=U_{i} \cap B .
$$

Thus $U_{1} \cap B=U_{2} \cap B$.

The proof is illustrated by the following "kite" diagram (the commuting fragments are marked by $\circlearrowright)$.



Proof of Theorem 3.2: Let $\mathbf{M}$ be an interior algebra and $\mathbf{N}$ be its subalgebra generated by all open elements in $\mathbf{M}$.

Assume first that $\mathbf{N}$ is a proper subalgebra of $\mathbf{M}$. Let $\mathbf{A}$ and $\mathbf{B}$ be Boolean reducts of $\mathbf{M}$ and $\mathbf{N}$ respectively. Let $U_{1}$ and $U_{2}$ be ultrafilters 
from Lemma 3.4. Let $\mathbf{S}$ be the simple interior algebras (only with 0 and 1 open) with the Boolean reduct $\mathbf{A} / U_{1} \times \mathbf{A} / U_{2}$. Then $\mathbf{S}$ is isomorphic to $\mathbf{S}_{2}$. Let $f: A \rightarrow S$ be given by $a \mapsto\left(a / U_{1}, a / U_{2}\right)$. Then $f$ is a surjective Boolean homomorphism. Moreover, since $U_{1} \cap N=U_{2} \cap N, f(N)=\{0,1\}$. Thus, by Lemma 2.1, $f$ is a stable homomorphism from $\mathbf{M}$ onto $\mathbf{S}$.

Now assume that $\mathbf{M}$ is generated by its open elements, i.e., $\mathbf{M}=\mathbf{N}$. Then $\mathbf{M}$ is also Boolean generated by its open elements. Let $f$ be any stable homomorphism from $\mathbf{M}$ into $\mathbf{S}_{2}$. By Lemma 2.1, for every $a \in M$ we have $f(\square a) \in\{0,1\}$. Since $f$ preserves Boolean operations and $\{0,1\}$ is closed under Boolean operations, $f$ maps $M$ onto $\{0,1\}$. Thus $f$ is not surjective.

EXAMPLE 3.5. There is a Grzegorczyk algebra that admits a stable homomorphism onto $\mathbf{S}_{2}$. Let us recall the following simple example $\mathbf{M}$. The carrier $M$ is the power set of the set $\mathbb{N}$ of natural numbers. The Boolean operations are the set theoretic operations. Let

$$
\square a=\left\{\begin{array}{ll}
1 & \text { if } a=1 \\
\{0, \ldots, k-1\} & \text { if } a \neq 1 \text { and } k=\min \neg a
\end{array} .\right.
$$

Note that $\mathbf{M}$ is the dual algebra for the modal frame $(\mathbb{N}, \geqslant)$ in the JónssonTarski duality. It is known that $\mathbf{M}$ is a Grzegorczyk algebra: The condition (Grz) holds for open $a$. So assume that $a$ is not open. Then $a-\square a \neq 0$ and the computation shows that

$$
\square(a \rightarrow \square a) \rightarrow a=\mathbb{N}-\{k, \ldots, l\},
$$

where $k=\min \neg a$ and $l+1=\min (a-\square a)$. This yields (Grz) for $a$.

Let $F$ be the Boolean filter of all cofinite subsets of $\mathbb{N}$. Let $\mathbf{S}$ be the simple interior algebra with the Boolean reduct obtained by dividing the Boolean reduct of $\mathbf{M}$ by $F$, and only two open elements 0 and 1 . Then $f: a \mapsto a / F$ is a stable homomorphism from $\mathbf{M}$ onto $\mathbf{S}$. Moreover, since $\mathbf{S}$ is infinite, it admits a stable homomorphism onto $\mathbf{S}_{2}$. Thus $\mathbf{M}$ admits a stable homomorphism onto $\mathbf{S}_{2}$.

ExAmple 3.6. There exists an interior algebra $\mathbf{M}$ which is not a Grzegorczyk algebra and which has no principal stable homomorphism $f$ onto any simple interior algebra with more than two elements. As the Boolean reduct of $\mathbf{M}$ we take the Boolean algebra of all subsets of $\mathbb{N}$ and as the 
collection of open elements in $\mathbf{M}$ we take $\left\{a_{k} \mid k \in \mathbb{N}\right\} \cup\{\emptyset\}$, where $a_{k}=\{k, k+1, \ldots\}$. The algebra $\mathbf{M}$ is the dual algebra for the modal frame $(\mathbb{N}, \leqslant)$ in the Jónsson-Tarski duality. In order to see that $\mathbf{M}$ is not a Grzegorczyk algebra, take $a \in M$ such that $a$ and $\neg a$ are both infinite. Then $a$ generates a subalgebra of $\mathbf{M}$ which is isomorphic to $\mathbf{S}_{2}$.

Now let $f: \mathbf{M} \rightarrow \mathbf{S}$ be any surjective principal stable homomorphism onto a simple interior algebras. Assume that the filter $f^{-1}(1)$ is generated by $d$. Then there is $k \in \mathbb{N}$ such that $f\left(a_{k}\right)=0$. Indeed, by Lemma 2.1 , if it is not the case, then $f\left(a_{k}\right)=1$ for all $k$. But it would yield that $d \leqslant a_{k}$ for all $k$, and hence $d=0$. This is a condradiction with the fact that $\mathbf{S}$, as a simple algebra, has at least two elements. Let $l=\min \left\{k \in \mathbb{N} \mid f\left(a_{k}\right)=0\right\}$. Since $a_{0}=1, l \geqslant 1$. We have that $f\left(a_{l-1}\right)=1$. Hence

$$
d \leqslant a_{l-1} \quad \text { and } \quad a_{l} \leqslant \neg d .
$$

This gives that $d \leqslant\{l-1\}$ and, since $f$ is surjective, $\mathbf{S}$ has at most two elements.

\section{Acknowledgments}

The proof of Theorem 3.2 was already published in [16]. We would like to thank the reviewer of [16] for indicating the relevant references [8, 12].

The work was supported by the Polish National Science Centre grant no. DEC- 2011/01/D/ST1/06136.

\section{References}

[1] G. Bezhanishvili and N. Bezhanishvili, An algebraic approach to canonical formulas: modal case, Studia Logica 99 (2011), pp. 93-125.

[2] G. Bezhanishvili, N. Bezhanishvili and R. Iemhoff, Stable canonical rules, Journal of Symbolic Logic 81 (2016), pp. 284-315.

[3] W. J. Blok, Varieties of interior algebras, $\mathrm{PhD}$ thesis, University of Amsterdam (1976), URL=http://www.illc.uva.nl/Research/Dissertations/HDS01-Wim_Blok.text.pdf.

[4] W. J. Blok and Ph. Dwinger, Equational classes of closure algebras. I, Indagationes Mathematicae 37 (1975), pp. 189-198.

[5] A. Chagrov and M. Zakharyaschev, Modal logic, Oxford University Press, New York, 1997.

[6] A. Chagrov and M. Zakharyashchev, Modal companions of intermediate propositional logics, Studia Logica 51 (1992), pp. 49-82. 
[7] L. L. Esakia, On the theory of modal and superintuitionistic systems, [in:] V. A. Smirnov (ed.), Logical inference, Nauka, Moscow (1979), pp. 147-172 (in Russian).

[8] L. L. Esakia, On the variety of Grzegorczyk algebras, [in:] Studies in nonclassical logics and set theory, Nauka, Moscow (1979), pp. 257-287 (in Russian).

[9] S. Ghilardi, Continuity, freeness, and filtrations, Journal of Applied NonClassical Logics 20 (2010), pp. 193-217.

[10] S. Givant and P. Halmos, Introduction to Boolean algebras, Springer, New York, 2009.

[11] A. Grzegorczyk, Some relational systems and the associated topological spaces, Fundamenta Mathematicae 60 (1967), pp. 223-231.

[12] D. C. Makinson, On the number of ultrafilters of an infinite boolean algebra, Zeitschrift fur mathematische Logik und Grundlagen der Mathematik 15 (1969), pp. 121-122.

[13] L. L. Maksimova and V. V. Rybakov, The lattice of normal modal logics, Algebra and Logic 13 (1974), pp. 105-122.

[14] J. C. C. McKinsey and A. Tarski, On closed elements in closure algebras, Annals of Mathematics 47 (1946), pp. 122-162.

[15] A. Y. Muravitsky, The embedding theorem: its further developments and consequences. Part I, Notre Dame Journal of Formal Logic 47 (2006), pp. $525-540$.

[16] M. M. Stronkowski, Free Boolean extensions of Heyting algebras, Advances in Modal Logic, Budapest, 2016, pp. 122-126 (Extended abstract).

[17] M. M. Stronkowski, On the Blok-Esakia theorem for universal classes, arXiv:1810.09286.

[18] F. Wolter and M. Zakharyaschev, On the Blok-Esakia theorem, [in:] G. Bezhanishvili (ed.), Leo Esakia on Duality in Modal and Intuitionistic Logics, Springer Netherlands, Dordrecht (2014), pp. 99-118.

[19] M. Zakharyaschev, Canonical formulas for K4. Part I. Basic results Journal of Symbolic Logic 57 (1992), pp. 1377-1402.

Faculty of Mathematics and Information Science

Warsaw University of Technology

ul. Koszykowa 75, 00-662 Warsaw, Poland

e-mail: m.stronkowski@mini.pw.edu.pl 